\title{
VIRTUALLY INDECOMPOSABLE TENSOR CATEGORIES
}

\author{
Shlomo GeLAKI
}

\begin{abstract}
Let $k$ be any field. J-P. Serre proved that the spectrum of the Grothendieck ring of the $k$-representation category of a group is connected, and that the same holds in characteristic zero for the representation category of a Lie algebra over $k$ [Se]. We say that a tensor category $\mathcal{C}$ over $k$ is virtually indecomposable if its Grothendieck ring contains no nontrivial central idempotents. We prove that the following tensor categories are virtually indecomposable: Tensor categories with the Chevalley property; representation categories of affine group schemes; representation categories of formal groups; representation categories of affine supergroup schemes (in characteristic $\neq 2$ ); representation categories of formal supergroups (in characteristic $\neq 2$ ); symmetric tensor categories of exponential growth in characteristic zero. In particular, we obtain an alternative proof to Serre's Theorem, deduce that the representation category of any Lie algebra over $k$ is virtually indecomposable also in positive characteristic (this answers a question of Serre [Se]), and (using a theorem of Deligne [D] in the super case, and a theorem of Deligne-Milne [DM] in the even case) deduce that any (super)Tannakian category is virtually indecomposable (this answers another question of Serre [Se]).
\end{abstract}

\section{Introduction}

The following theorem is due to J-P. Serre.

Theorem 1.1. [Se, Corollary 5.5 \& Section 5.1.2; Ex. 3] Let $k$ be a field.

(1) Let $G$ be any group, let $\operatorname{Rep}(G)$ be the category of finite-dimensional representations of $G$ over $k$, and let $G r(G)$ be its (commutative) Grothendieck ring. Then the spectrum $\operatorname{Spec}(G r(G))$ of $G r(G)$ is connected.

(2) Assume that $k$ has characteristic zero. Let $\mathfrak{g}$ be a Lie algebra over $k$, let Rep $(\mathfrak{g})$ be the category of finite-dimensional representations of $\mathfrak{g}$ over $k$, and let $G r(\mathfrak{g})$ be its (commutative) Grothendieck ring. Then $\operatorname{Spec}(\operatorname{Gr}(\mathfrak{g}))$ is connected.

The proof of Theorem 1.1 uses, among other things, the fact that the semisimple representations of a group $G$ are detected by their characters, in characteristic zero, and by their Brauer characters, in positive characteristic.

Recall that the category $\operatorname{Rep}(G)$ is an example of a Tannakian category [DM] (see Section 2). Motivated by Theorem 1.1 and this fact, Serre asked the following question.

Question 1.1. [Se, Section 5.1.2; Ex. 4] Let $\mathcal{C}$ be a Tannakian category over any field $k$ and let $\operatorname{Gr}(\mathcal{C})$ be its Grothendieck ring. Is it true that $\operatorname{Spec}(G r(\mathcal{C}))$ is connected? In particular, let $\mathfrak{g}$ be a Lie algebra over any field $k$ and let $\mathcal{C}:=\operatorname{Rep}(\mathfrak{g})$ be the

Received by the editors September 10, 2011.

Key words and phrases. tensor category, Grothendieck ring, Hopf (super)algebra, affine (super)group scheme, formal (super)group. 
category of finite-dimensional representations of $\mathfrak{g}$ over $k$. Is it true that $\operatorname{Spec}(\operatorname{Gr}(\mathfrak{g}))$ is connected?

Question 1.1 can be extended to any tensor category over $k$, namely to a $k$-linear locally finite abelian category with finite-dimensional Hom-spaces, equipped with an associative tensor product and unit. (See, e.g., [E] for the definition of a tensor category and its general theory.)

Definition 1.1. Let $k$ be any field, and let $\mathcal{C}$ be any tensor category over $k$. Let $R$ be any commutative ring. We say that $\mathcal{C}$ is virtually indecomposable over $R$ if its Grothendieck ring $R \otimes_{\mathbb{Z}} G r(\mathcal{C})$ with $R$-coefficients has no nontrivial central idempotents, and that $\mathcal{C}$ is strongly virtually indecomposable over $R$ if $R \otimes_{\mathbb{Z}} G r(\mathcal{C})$ has no nontrivial idempotents. In the case $R=\mathbb{Z}$ we shall suppress the phrase "over $\mathbb{Z}$ ".

Question 1.2. Is it true that any tensor category over any field is virtually indecomposable? Strongly virtually indecomposable?

Our goal in this paper is to provide a positive answer to Question 1.2 for a variety of tensor categories over any field $k$. More precisely, we prove that the following tensor categories are virtually indecomposable:

- Tensor categories with the Chevalley property.

- Representation categories of affine group schemes.

- Representation categories of formal groups.

- Representation categories of affine supergroup schemes (in characteristic $\neq 2$ ).

- Representation categories of formal supergroups (in characteristic $\neq 2$ ).

- Symmetric tensor categories of exponential growth in characteristic zero.

In particular, we obtain both an alternative proof to Theorem 1.1 and a positive answer to Question 1.1.

\section{The main results}

The following standard lemma shows that without loss of generality we may (and shall) work over an algebraically closed field.

Lemma 2.1. If $\mathcal{C}$ is a locally finite abelian category over a field $k$ then the map $\operatorname{Gr}(\mathcal{C}) \rightarrow \operatorname{Gr}\left(\mathcal{C} \otimes_{k} \bar{k}\right)$ is injective.

Proof. It is well known that $\mathcal{C}$ is equivalent to the category of finite-dimensional $A$ comodules over $k$, where $A$ is a coalgebra over $k$. Let us denote $\operatorname{Gr}(\mathcal{C})$ by $\operatorname{Gr}(A)$. We need to show that the map $\operatorname{Gr}(A) \rightarrow G r\left(A \otimes_{k} \bar{k}\right)$ is injective. Clearly, we may assume that $A$ is finite dimensional, so $\mathcal{C}=\operatorname{Rep}\left(A^{*}\right)$. Then we can pass to the quotient of $A^{*}$ by its radical and assume that $A^{*}$ is semisimple. So we can assume that $A^{*}$ is simple, i.e., $A^{*}=\operatorname{Mat}_{n}(D), D$ a division algebra over $k$. But in this case the claim is obvious since $\operatorname{Gr}(A)=\mathbb{Z}$.

Corollary 2.1. A tensor category $\mathcal{C}$ over $k$ is virtually indecomposable if $\mathcal{C} \otimes_{k} \bar{k}$ is virtually indecomposable. 
Therefore, throughout the paper we shall work over an algebraically closed field $k$.

2.1. Based rings. In Section 3.1, we recall the definition of a unital-based ring, and then prove in Section 3.2 the following theorem about them.

Theorem 2.1. Let $A$ be any unital based ring. Then $A$ is virtually indecomposable.

Recall that a $k$-linear abelian rigid tensor category $\mathcal{C}$ is said to have the Chevalley property if the tensor product of any two semisimple objects of $\mathcal{C}$ is also semisimple. In other words, the subcategory $\mathcal{C}_{s s}$ of semisimple objects in $\mathcal{C}$ is a tensor subcategory. For example, in characteristic zero, $\mathcal{C}=\operatorname{Rep}(G)$ and $\mathcal{C}=\operatorname{Rep}(\mathfrak{g})$, where $G$ is any group and $\mathfrak{g}$ is any Lie algebra, have the Chevalley property $[\mathrm{C}]$. Of course, if $\mathcal{C}$ is semisimple (e.g., a fusion category) then $\mathcal{C}$ has the Chevalley property.

Now, if $\mathcal{C}$ has the Chevalley property then $\operatorname{Gr}(\mathcal{C})=\operatorname{Gr}\left(\mathcal{C}_{s s}\right)$, so $\operatorname{Gr}(\mathcal{C})$ is a unital based ring. Hence, Theorem 2.1 implies the following corollary.

Corollary 2.2. Let $\mathcal{C}$ be a $k$-linear abelian rigid tensor category. If $\mathcal{C}$ has the Chevalley property then $\mathcal{C}$ is virtually indecomposable.

Remark 2.1. In general, it is not true that the representation categories of groups and Lie algebras in positive characteristic have the Chevalley property, and likewise for supergroups and Lie superalgebras in any characteristic.

2.2. The Hopf algebra case. In Section 4, we prove the following innocent looking result, which will turn out to play the key role in proving our results concerning (super)groups and (super)Lie algebras.

Theorem 2.2. Let $H$ be a (not necessarily commutative) Hopf algebra over a field $k$, and let $\operatorname{Corep}(H)$ denote the tensor category of finite-dimensional $H$-comodules over $k$. Suppose that $I$ is a Hopf ideal in $H$ such that $\bigcap_{n \geq 1} I^{n}=0$. Let $R$ be any commutative ring and, if the characteristic of $k$ is $p>0$, assume that $\bigcap_{n>1} p^{n} R=0$. Then, if $\operatorname{Corep}(H / I)$ is virtually indecomposable over $R$ then so is Corep $(H)$.

Remark 2.2. In fact, Theorem 2.2 holds also, with the same proof, in the topological case (i.e., when $H$ is a topological Hopf algebra; see below).

2.3. The group case. In Section 5 , we use Theorem 2.2 to prove increasingly strong results, culminating in the following theorem.

Theorem 2.3. Let $k$ be any field, and let $G$ be an affine group scheme over $k$. Let $S$ be the set of all primes not equal to the characteristic of $k$ and not dividing $\left|G / G^{0}\right|$. Then $\operatorname{Spec}\left(\mathbb{Z}\left[S^{-1}\right] \otimes_{\mathbb{Z}} \operatorname{Gr}(G)\right)$ is connected.

Theorem 2.3 generalizes to formal groups. Recall that a formal group $G$ over a field $k$, whose subset of closed points (= reduced part) is the affine proalgebraic group $\bar{G}$ over $k$, is the following algebraic structure. We have a structure algebra $\mathcal{O}(G)$ over $k$, which has an ideal $I$ such that $\mathcal{O}(G) / I=\mathcal{O}(\bar{G})$, and $\mathcal{O}(G)$ is complete and separated in the topology defined by $I$ (i.e., $\mathcal{O}(G)=\underline{\lim } \mathcal{O}(G) / I^{m}$ ). Finally, we have 
a cocommutative coproduct $\Delta: \mathcal{O}(G) \rightarrow \mathcal{O}(G) \widehat{\otimes} \mathcal{O}(G)$, where the latter completed tensor product is $\lim _{(}\left(\mathcal{O}(G) / I^{m} \otimes \mathcal{O}(G) / I^{m}\right)$, defining a topological Hopf algebra structure on $\mathcal{O}(G)$, such that $I$ is a Hopf ideal, and the isomorphism $\mathcal{O}(G) / I \rightarrow \mathcal{O}(\bar{G})$ is a Hopf algebra isomorphism.

Thus, combining Theorems 2.2 and 2.3, we obtain the following result.

Theorem 2.4. Let $k$ be any field, and let $G$ be a formal group over $k$ with reduced part $\bar{G}$. Let $S$ be the set of all primes not equal to the characteristic of $k$ and not dividing $\left|\bar{G} / \bar{G}^{0}\right|$. Then $\operatorname{Spec}\left(\mathbb{Z}\left[S^{-1}\right] \otimes_{\mathbb{Z}} G r(G)\right)$ is connected.

Therefore, as an immediate corollary of Theorem 2.4 (the case $\bar{G}=1$ ), we deduce a positive answer to the second part of Serre's Question 1.1. Nevertheless, in Section 4.3 we shall also give a self-contained proof of this theorem in the positive characteristic case.

Theorem 2.5. Let $\mathfrak{g}$ be a Lie algebra over any field $k$ and let $\mathcal{C}:=\operatorname{Rep}(\mathfrak{g})$ be the category of finite-dimensional representations of $\mathfrak{g}$ over $k$. Then $\operatorname{Spec}(G r(\mathfrak{g}))$ is connected.

Remark 2.3. Note that the case $\bar{G}=1$ (formal groups with one closed point) reduces to Lie algebras in characteristic zero, but in positive characteristic it contains much more.

Recall that a Hopf algebra $H$ over a field $k$ is called coconnected if every simple $H$-comodule over $k$ is trivial (see e.g. [EG] where, in particular, coconnected Hopf algebras over $\mathbb{C}$ are classified in Theorem 4.2). We have the following result which extends Theorem 2.5.

Theorem 2.6. Let $H$ be a coconnected Hopf algebra over any field $k$, and let $S$ be the set of all primes not equal to the characteristic of $k$. Then $R e p(H)$ is virtually indecomposable over $\mathbb{Z}\left[S^{-1}\right]$.

Proof. If $H$ is coconnected then $H^{*}$ is a topological Hopf algebra with maximal ideal $I:=\operatorname{Ker}(\epsilon)$, which is complete and separated in the topology defined by $I$ (as the powers of $I$ are orthogonal to the terms of the coradical filtration of $H$ ). So the claim follows from the topological version of Theorem 2.2 (see Remark 2.2).

2.4. The supergroup case. In Section 6.1, we recall the notion of a Hopf superalgebra, and in Section 6.2, we recall the notions of an affine supergroup scheme and a formal supergroup over $k$. We then generalize in Section 6.3 the results from Section 5 to the super-case (assuming the characteristic of $k \neq 2$ ).

Let $\mathcal{G}$ be an affine supergroup scheme or, more generally, a formal supergroup, and let $u \in \mathcal{G}$ be an element of order 2 acting by parity on the algebra of regular functions $\mathcal{O}(\mathcal{G})$. Let $\operatorname{Rep}(\mathcal{G}, u)$ be the category of representations of $\mathcal{G}$ on finitedimensional supervector spaces over $k$ on which $u$ acts by parity, and let $\operatorname{Gr}(\mathcal{G}, u)$ be its Grothendieck ring.

Theorem 2.7. Let $k$ be any field of characteristic $\neq 2$. Let $\mathcal{G}$ be an affine supergroup scheme over $k$ or, more generally, a formal supergroup over $k$. Let $S$ be the set of all primes $\neq 2$ not equal to the characteristic of $k$ and not dividing $\left|\mathcal{G} / \mathcal{G}^{0}\right|$. Then $\operatorname{Spec}\left(\mathbb{Z}\left[S^{-1}\right] \otimes_{\mathbb{Z}} \operatorname{Gr}(\mathcal{G}, u)\right)$ is connected. 
Remark 2.4. Note that the prime 2 must be excluded (i.e., cannot be inverted). Indeed, already in the category SuperVect of finite-dimensional supervector spaces over $k$ (see Section 6 ), the element $\frac{1}{2}\left(k_{0} \oplus k_{1}\right)$ is a nontrivial idempotent.

Recall that a Lie superalgebra over a field $k$ is a Lie algebra in SuperVect (see e.g, $[\mathrm{B}]$ ). In other words, a Lie superalgebra $\mathfrak{g}=\mathfrak{g}_{0} \oplus \mathfrak{g}_{1}$ is a supervector space over $k$, equipped with an operation [,] $: \mathfrak{g} \otimes \mathfrak{g} \rightarrow \mathfrak{g}$ satisfying the following axioms: $[x, y]=-(-1)^{|x||y|}[y, x]$ and $[x,[y, z]]=[[x, y], z]+(-1)^{|x||y|}[y,[x, z]]$, for homogeneous elements $x, y \in \mathfrak{g}$ and $z \in \mathfrak{g}$. The following result on Lie superalgebras is an immediate corollary of Theorem 2.7.

Corollary 2.3. Let $\mathfrak{g}$ be a Lie superalgebra over a field $k$ of characteristic $\neq 2$. Let $S$ be the set of all primes $\neq 2$ not equal to the characteristic of $k$. Then the spectrum of $\mathbb{Z}\left[S^{-1}\right] \otimes_{\mathbb{Z}} G r(\mathfrak{g})$ is connected.

By a theorem of Deligne $[\mathrm{D}]$ in characteristic zero, the categories $\operatorname{Rep}(\mathcal{G}, u)$ exhaust all $k$-linear abelian symmetric rigid tensor categories of exponential growth. Hence, we deduce the following corollary.

Corollary 2.4. If $\mathcal{C}$ is a $k$-linear abelian symmetric rigid tensor category of exponential growth over an algebraically closed field $k$ ofcharacteristic zero, then $\mathcal{C}$ is virtually indecomposable.

Recall that a (super)Tannakian category over a field $k$ is a $k$-linear abelian symmetric rigid tensor category $\mathcal{C}$, with $\operatorname{End}(\mathbf{1})=k$, where $\mathbf{1}$ denotes the unit object, which admits a fiber functor to the category of finite-dimensional (super)vector spaces (see $[\mathrm{D}]$ ). In the following proposition we deduce a positive answer to the first part of Serre's Question 1.1.

Proposition 2.1. A (super)Tannakian category $\mathcal{C}$ over any field $k$ is virtually indecomposable.

Proof. By (the super analog of) a theorem of Deligne-Milne [DM] (which is in [D]), $\mathcal{C}$ is equivalent to a category of the form $\operatorname{Rep}(\mathcal{G}, u)$, so the claim follows by Theorem 2.7.

\section{The virtually indecomposability of a unital based ring}

In characteristic zero there is an alternative ("combinatorial") proof of (a slight generalization of) Theorem 1.1 in the framework of unital-based rings.

3.1. Based rings. Let $A$ be a ring with a distinguished $\mathbb{Z}$-basis $\left\{b_{i}\right\}, i \in I$, (not necessarily of finite rank), which contains the unit element 1 , such that $b_{i} b_{j}=\sum_{k} n_{i j}^{k} b_{k}$, where $n_{i j}^{k} \in \mathbb{Z}^{+}$. The bilinear map $\left(\sum_{i} n_{i} b_{i}, \sum_{i} m_{i} b_{i}\right) \mapsto \sum_{i} n_{i} m_{i}$ defines a positive inner product (, ) : $A \times A \rightarrow \mathbb{Z}$ on $A$. We call $A$ a unital based ring if there is an involution $i \mapsto i^{*}$ such that the induced map $x=\sum_{i} n_{i} b_{i} \mapsto x^{*}:=\sum_{i} n_{i} b_{i^{*}}$ satisfies $(x y, z)=\left(x, z y^{*}\right)=\left(y, x^{*} z\right)$ for all $x, y, z \in A$. In particular, it follows that the matrix of multiplication by $x^{*}$ is transposed to the matrix of multiplication by $x$, for any $x \in A$. 
Example 3.1. If $\mathcal{C}$ is a $k$-linear semisimple rigid tensor category, its Grothendieck ring $\operatorname{Gr}(\mathcal{C})$ is a unital-based ring. A typical example of such category is the category $\mathcal{C}:=\operatorname{Corep}(H)$ of finite-dimensional comodules of a cosemisimple Hopf algebra $H$. The distinguished $\mathbb{Z}$-basis of $G r(\mathcal{C})$ consists of the isomorphism classes of simple $H$ comodules, and the involution $*$ is given by taking the $k$-linear dual of a comodule.

3.2. The proof of Theorem 2.1. Let $e \neq 1$ be a central idempotent in $A$. We have to show that $e=0$. We first note that $e$ is a projection operator on an inner product space, which is normal (i.e., $e e^{*}=e^{*} e$ ), so $e$ is self-adjoint. Indeed, $\left(e\left(1-e^{*}\right), e\left(1-e^{*}\right)\right)=\left(e^{*} e\left(1-e^{*}\right), 1-e^{*}\right)=\left(e e^{*}\left(1-e^{*}\right), 1-e^{*}\right)=0$. Thus, by positivity of the inner product, $e\left(1-e^{*}\right)=0$, so $e=e e^{*}$, hence $e=e^{*}$.

Then $e$ is an orthogonal projector to a proper subspace of $\mathbb{R} \otimes_{\mathbb{Z}} A$, which does not contain 1 . So $0 \leq(e, e)=(e 1, e 1)<(1,1)=1$. But $(e, e)$ is an integer, so $(e, e)=0$, and hence $e=0$.

Remark 3.1. It is interesting to mention here a classical result of Kaplansky which asserts that there is no nontrivial idempotent in the integral group ring of any (not necessarily commutative) group (see $[\mathrm{K}],[\mathrm{P}])$, i.e., the integral group ring of any group is strongly virtually indecomposable. Equivalently, the tensor category $V e c_{G}$ of $G$ graded vector spaces over $k$ is strongly virtually indecomposable for any groupz $G$.

In fact, Proposition 3, in [R] extends the result of Kaplansky to fusion rings (= unital based rings of finite rank). Equivalently, any fusion category is strongly virtually indecomposable.

\section{The proof of Theorem 2.2.}

In this section, we let $H$ be a Hopf algebra (not necessarily commutative) over $k$, and $\mathcal{C}:=\operatorname{Corep}(H)$ be the category of finite-dimensional right comodules of $H$. Then $\mathcal{C}$ is a $k$-linear abelian rigid tensor category in which every object has a finite length. Let $\operatorname{Gr}(\mathcal{C})$ be the Grothendieck ring of $\mathcal{C}$; it is the free $\mathbb{Z}$-algebra with a distinguished basis formed by the classes $[X]$ of the simple objects $X \in \mathcal{C}$.

4.1. Characters in Hopf algebras. Recall that any $M \in \mathcal{C}$ has a canonical rational $H^{*}$-module structure.

Definition 4.1. For an object $M \in \mathcal{C}$, the character $\operatorname{ch}(M)$ of $M$ is the character of the $H^{*}$-module $M$. In other words, the character $\operatorname{ch}(M)$ is the function $H^{*} \rightarrow k$ defined by $\operatorname{ch}(M)(x):=\operatorname{tr}\left(x_{\mid M}\right)$.

Clearly, $\operatorname{ch}(M) \in H, \operatorname{ch}(M) \operatorname{ch}(N)=\operatorname{ch}(M \otimes N)$ and $\operatorname{ch}(M)+\operatorname{ch}(N)=\operatorname{ch}(M \oplus N)$. Moreover, if $M_{1}, \ldots, M_{n}$ are the distinct composition factors of $M$, with multiplicities $a_{1}, \ldots, a_{n}$, then $\operatorname{ch}(M)=\sum_{i=1}^{n} a_{i} c h\left(M_{i}\right)$. In other words, the character of $M$ and the character of its semisimplification $\bigoplus_{i=1}^{n} a_{i} M_{i}$ coincide. We therefore have a welldefined $k$-algebra homomorphism

$$
\operatorname{ch}: k \otimes_{\mathbb{Z}} \operatorname{Gr}(\mathcal{C}) \rightarrow H, a \otimes[M] \mapsto a \cdot \operatorname{ch}(M) .
$$

Proposition 4.1. The character map ch is injective. In other words, if $M, N \in \mathcal{C}$ with $\operatorname{ch}(M)=\operatorname{ch}(N)$, then $[M]=[N]$ in $k \otimes_{\mathbb{Z}} G r(\mathcal{C})$. 
Proof. It is enough to show that if $\sum_{i=1}^{m} a_{i} c h\left(M_{i}\right)=0$ on $H^{*}$, for some finite number of nonisomorphic irreducible comodules $M_{i} \in \mathcal{C}$ and some elements $a_{i} \in k$, then $a_{i}=0$ for all $i$.

Indeed, by the density theorem, the map $H^{*} \rightarrow \oplus_{i} \operatorname{End}_{k}\left(M_{i}\right)$ is surjective, so we can choose an element $x \in H^{*}$ which maps to 0 on $\operatorname{End}_{k}\left(M_{j}\right)$ for $j \neq i$, and to an element with trace 1 on $\operatorname{End}_{k}\left(M_{i}\right)$, which implies that $a_{i}=0$ for all $i$.

Remark 4.1. Note that if the characteristic of $k$ is zero then Proposition 4.1 implies that the character of $M$ determines the composition factors of $M$ together with their multiplicities, i.e., $c h: G r(\mathcal{C}) \rightarrow H$ is injective (so in particular, if $M, N$ are semisimple then $M, N$ are isomorphic). On the other hand, if the characteristic of $k$ is $p>0$ then Proposition 4.1 implies only that the character of $M$ determines the composition factors of $M$ together with their multiplicities modulo $p$.

4.2. The proof of Theorem 2.2. Set $\overline{\mathcal{C}}:=\operatorname{Corep}(H / I)$. The surjection of Hopf algebras $H \rightarrow H / I$ induces a tensor functor $\mathcal{C} \rightarrow \overline{\mathcal{C}}$, which in turn induces a ring homomorphism $R \otimes_{\mathbb{Z}} G r(\mathcal{C}) \rightarrow R \otimes_{\mathbb{Z}} G r(\overline{\mathcal{C}})$. Suppose $E \in R \otimes_{\mathbb{Z}} G r(\mathcal{C})$ is an idempotent which is not 0 or 1 , and let $e$ be the image of $E$ in $R \otimes_{\mathbb{Z}} G r(\overline{\mathcal{C}})$. By assumption, $e$ is either 0 or 1 . Without loss of generality we may assume that $e=0$, replacing $E$ by $1-E$ if needed.

Now, if $k$ has characteristic $p>0$, at least one of the coefficients of $E$ is not divisible by $p$. Indeed, if $E=p F$ then $E^{n}=p^{n} F^{n}=E$, so $E \in p^{n} R \otimes_{\mathbb{Z}} G r(\mathcal{C})$ for all $n$, and hence it is zero, which is a contradiction. Therefore, the image $E^{\prime}$ of $E$ in $R \otimes_{\mathbb{Z}} k \otimes_{\mathbb{Z}} G r(\mathcal{C})$ (which is $(R / p R) \otimes_{\mathbb{F}_{p}} k \otimes_{\mathbb{Z}} G r(\mathcal{C})$ in positive characteristic) is nonzero, and the image $e^{\prime}$ of $e$ in $R \otimes_{\mathbb{Z}} k \otimes_{\mathbb{Z}} G r(\overline{\mathcal{C}})$ is zero (as $e=0$ ).

Now, using the embedding $c h: k \otimes_{\mathbb{Z}} \operatorname{Gr}(\mathcal{C}) \hookrightarrow H$, we get a nonzero idempotent $\operatorname{ch}\left(E^{\prime}\right)$ in $R \otimes_{\mathbb{Z}} H$, which has zero image in $R \otimes_{\mathbb{Z}} H / I$ (this image is $\operatorname{ch}\left(e^{\prime}\right)$ ). This implies that $\operatorname{ch}\left(E^{\prime}\right) \in R \otimes_{\mathbb{Z}} I$. But since $\operatorname{ch}\left(E^{\prime}\right)$ is an idempotent, $\operatorname{ch}\left(E^{\prime}\right)^{n}=\operatorname{ch}\left(E^{\prime}\right)$ for all $n$, so $\operatorname{ch}\left(E^{\prime}\right) \in \bigcap_{n \geq 1}\left(R \otimes_{\mathbb{Z}} I^{n}\right)=0$, which is a contradiction.

4.3. A proof of Theorem 2.5. Let $\mathfrak{g}$ be a Lie algebra over a field $k$ of characteristic $p>0$; we may assume without loss of generality that $k$ is algebraically closed (see Corollary 2.1). Let $A:=U(\mathfrak{g})^{*}$ be the dual algebra of the universal enveloping algebra $U(\mathfrak{g})$ of $\mathfrak{g}$ (it is a topological Hopf algebra in the topology defined by the maximal ideal $I$ of $A)$. Let $E \in G r(\mathfrak{g})$ be an idempotent which is not 0 or 1 . We can assume that $\operatorname{Tr}_{E}(1)=0$ modulo $p$ by replacing $E$ with $1-E$ if needed.

Now, at least one of the coefficients of $E$ is not divisible by $p$. Indeed, otherwise $(E / p)^{n}=E / p^{n}$, so $E / p^{n} \in G r(\mathfrak{g})$ for all $n$, but $E / p^{n}$ does not have integer coefficients for large enough $n$. Consequently, the image $E^{\prime}$ of $E$ in $k \otimes_{\mathbb{Z}} G r(\mathfrak{g})$ is nonzero. Hence, using Proposition 4.1, we get a nonzero idempotent $\operatorname{ch}\left(E^{\prime}\right)$ in $A$. On the other hand, the augmentation map $A \rightarrow k$ maps $c h\left(E^{\prime}\right)$ to zero (since $\operatorname{Tr}_{E}(1)=0$ modulo $p$ ). So $\operatorname{ch}\left(E^{\prime}\right)$ is contained in $I$. But $\operatorname{ch}\left(E^{\prime}\right)$ is an idempotent, so it is contained in any power $I^{n}$ of $I$. But $\bigcap_{n \geq 1} I^{n}=0$, so $\operatorname{ch}\left(E^{\prime}\right)$ is zero, which is a contradiction.

\section{The proof of Theorem 2.3}

The proof of Theorem 2.3 will be carried in several steps. 
5.1. $G$ is a reductive abelian affine algebraic group over $k$. Recall that if $G$ is a reductive abelian affine algebraic group over $k$ then $G \cong G^{0} \times A$, where $G^{0}=\mathbb{G}_{m}^{n}$ is the $n$-dimensional torus over $k$ and $A$ is a finite abelian group of order prime to $p$ (in case the characteristic of $k$ is $p>0$ ) (see, e.g., [Sp]). In particular, all finite-dimensional simple representations of $G$ over $k$ are 1-dimensional, and their isomorphism types are parameterized by pairs $(z, \chi)$, where $z \in \mathbb{Z}^{n}$ and $\chi \in \widehat{A}$. Thus, all idempotents in $\mathbb{Q} \otimes_{\mathbb{Z}} G r(G)$ can be easily described in this case (they involve a factor of $1 /|A|)$, so the result follows in a straightforward manner.

5.2. $G$ is any abelian affine algebraic group over $\boldsymbol{k}$. Recall that if $G$ is an abelian affine algebraic group over $k$ then $G \cong G_{s} \times G_{u}$, where $G_{s}$ and $G_{u}$ are the subgroups of semisimple and unipotent elements of $G$, respectively (see, e.g., [Sp]). So $\operatorname{Rep}(G)$ and $\operatorname{Rep}\left(G_{s}\right)$ have the same Grothendieck rings, and the claim follows from 5.1.

5.3. $G$ is any affine algebraic group over $\boldsymbol{k}$. Let $G$ be any affine algebraic group over $k$, and let $\mathcal{O}(G)$ be its coordinate Hopf algebra; it is a finitely generated commutative reduced Hopf algebra over $k$. Suppose $e \in \operatorname{Gr}(\operatorname{Rep}(G))=\operatorname{Gr}(\operatorname{Corep}(\mathcal{O}(G)))$ is an idempotent. Then $c h(e) \in \mathcal{O}(G)$ is an idempotent, so in particular a class function on $G$ taking the values 0,1 (here we are using the trick with dividing by $p$, as we did in the proof of Theorem 2.2, which explains why we cannot invert $p$ ). Therefore, it suffices to prove that $\operatorname{ch}(e)(g)=\operatorname{ch}(e)(1)$ for all $g \in G$. But for that purpose we may assume that $G$ is the (Zariski closure of the) cyclic group with generator $g$, so $G$ is abelian and the claim follows from Section 5.2.

5.4. $G$ is an affine proalgebraic group over $\boldsymbol{k}$. Let $G$ be an affine proalgebraic group over $k$, and let $\mathcal{O}(G)$ be its coordinate Hopf algebra over $k$; it is a commutative reduced Hopf algebra over $k$ (not necessarily finitely generated). But it is well known that $\mathcal{O}(G)$ is the inductive limit of its finitely generated Hopf subalgebras (see e.g., $[\mathrm{A}])$, so the claim follows in a straightforward manner from Section 5.3.

5.5. $G$ is an affine group scheme over $k$. Let $G$ be an affine group scheme, and let $\mathcal{O}(G)$ be the commutative Hopf algebra representing the group functor $G$ (it is not necessarily reduced) (see, e.g., [W]). Since $k$ is algebraically closed, it is well known (essentially by Hilbert Nullstellensatz) that the nilradical $I$ of $\mathcal{O}(G)$ is a Hopf ideal. Now, the commutative reduced Hopf algebra $\mathcal{O}(G) / I$ over $k$ represents an affine proalgebraic group over $k$, so by Section 5.4, $\operatorname{Corep}(\mathcal{O}(G) / I)$ is virtually indecomposable. Finally, since $\bigcap_{n \geq 1} I^{n}=0$, it follows from Theorem 2.2 that $\operatorname{Corep}(\mathcal{O}(G))=\operatorname{Rep}(G)$ is virtually indecomposable, as claimed.

This concludes the proof of Theorem 2.3.

\section{The proof of Theorem 2.7}

6.1. Hopf superalgebras. Recall that a Hopf superalgebra over a field $k$ is a Hopf algebra in the $k$-linear abelian symmetric tensor category SuperVect of supervector spaces over $k$ (see, e.g, [B]). In other words, a Hopf superalgebra $\mathcal{H}=\mathcal{H}_{0} \oplus \mathcal{H}_{1}$ is an ordinary $\mathbb{Z}_{2}$-graded associative unital algebra over $k$ (i.e., a superalgebra), equipped with a coassociative morphism $\Delta: \mathcal{H} \rightarrow \mathcal{H} \otimes \mathcal{H}$ in SuperVect, which is multiplicative 
in the super-sense, and with a counit and antipode satisfying the standard axioms. Here multiplicativity in the super-sense means that $\Delta$ satisfies the relation

$$
\Delta(a b)=\sum(-1)^{\left|a_{2}\right|\left|b_{1}\right|} a_{1} b_{1} \otimes a_{2} b_{2},
$$

where $a, b \in \mathcal{H}$ are homogeneous elements, $|a|$ and $|b|$ denote the degrees of $a$ and $b$, $\Delta(a)=\sum a_{1} \otimes a_{2}$ and $\Delta(b)=\sum b_{1} \otimes b_{2}$.

A Hopf superalgebra $\mathcal{H}$ is said to be commutative if $a b=(-1)^{|a||b|} b a$ for all homogeneous elements $a, b \in \mathcal{H}$.

Let $J(\mathcal{H}):=\left(\mathcal{H}_{1}\right)=\mathcal{H}_{1}^{2} \oplus \mathcal{H}_{1}$ be the Hopf ideal of $\mathcal{H}$ generated by the odd elements $\mathcal{H}_{1}$. Then the quotient $\overline{\mathcal{H}}:=\mathcal{H} / J(\mathcal{H})$ is an ordinary Hopf algebra. Note that if $\mathcal{H}$ is commutative then $J(\mathcal{H})$ consists of nilpotent elements.

Let us recall the following useful construction, introduced in Section 3.1 in [AEG]. Let $\mathcal{H}$ be any Hopf superalgebra over $k$, and let $\widetilde{\mathcal{H}}:=k\left[\mathbb{Z}_{2}\right] \ltimes \mathcal{H}$ be the semidirect product Hopf superalgebra with comultiplication $\widetilde{\Delta}$ and antipode $\widetilde{S}$, where the generator $g$ of $\mathbb{Z}_{2}$ acts on $\mathcal{H}$ by $g h g^{-1}=(-1)^{|h|} h$. For $x \in \widetilde{\mathcal{H}}$, write $\widetilde{\Delta}(x)=\widetilde{\Delta}_{0}(x)+\widetilde{\Delta}_{1}(x)$, where $\widetilde{\Delta}_{0}(x) \in \widetilde{\mathcal{H}} \otimes \widetilde{\mathcal{H}}_{0}$ and $\widetilde{\Delta}_{1}(x) \in \widetilde{\mathcal{H}} \otimes \widetilde{\mathcal{H}}_{1}$. Then one can define an ordinary Hopf algebra structure on the algebra $\mathcal{H}^{\prime}:=\widetilde{\mathcal{H}}$, with comultiplication and antipode maps given by $\Delta(h):=\widetilde{\Delta}_{0}(h)-(-1)^{|h|}(g \otimes 1) \widetilde{\Delta}_{1}(h)$ and $S(h):=g^{|h|} \widetilde{S}(h), h \in \mathcal{H}^{\prime}$ (see Theorem 3.1.1 in $[\mathrm{AEG}])$.

Theorem 6.1. Let $\mathcal{H}$ be a Hopf superalgebra over a field $k$ of characteristic $\neq 2$, and let $\operatorname{Corep}(\mathcal{H})$ be the tensor category of finite-dimensional $\mathcal{H}$-comodules in SuperVect over $k$. The tensor categories $\operatorname{Corep}(\mathcal{H})$ and $\operatorname{Corep}\left(\mathcal{H}^{\prime}\right)$ are equivalent.

Proof. An $\mathcal{H}$-comodule in SuperVect is a (continuous) $\mathbb{Z}_{2}$-graded $\mathcal{H}^{*}$-module in the category Vect of vector spaces over $k$, which is the same as a continuous module over the algebra $k\left[\mathbb{Z}_{2}\right] \ltimes \mathcal{H}^{*}$. But $k\left[\mathbb{Z}_{2}\right] \ltimes \mathcal{H}^{*}$ is isomorphic to $\left(\mathcal{H}^{\prime}\right)^{*}$ as an algebra, so a $\mathcal{H}$-comodule in SuperVect is the same thing as a $\mathcal{H}^{\prime}$-comodule in Vect. Finally, it is easy to check that this equivalence is in fact a tensor equivalence.

We note that as a consequence of Theorem 6.1 , we can define the character map ch $: k \otimes_{\mathbb{Z}} \operatorname{Gr}(\operatorname{Corep}(\mathcal{H})) \rightarrow \mathcal{H}^{\prime}, a \otimes[M] \mapsto a \cdot \operatorname{ch}(M)$, and deduce from Proposition 4.1 that it is an injective $k$-algebra homomorphism.

6.2. Affine supergroup schemes and formal supergroups. Recall that an affine supergroup scheme $\mathcal{G}$ is the spectrum of a (not necessarily finitely generated) commutative Hopf superalgebra $\mathcal{O}(\mathcal{G})$ over $k$ (see, e.g., [D]). In other words, it is a functor $\mathcal{G}$ from the category of supercommutative algebras to the category of groups defined by $A \mapsto \mathcal{G}(A):=\operatorname{Hom}(\mathcal{O}(\mathcal{G}), A)$, where $\operatorname{Hom}(\mathcal{O}(\mathcal{G}), A)$ is the group of algebra maps $\mathcal{O}(\mathcal{G}) \rightarrow A$ in SuperVect.

Recall that a formal supergroup $\mathcal{G}$ over a field $k$, with reduced part $G$, is the following algebraic structure. We have a superalgebra $\mathcal{O}(\mathcal{G})$ over $k$, which has an ideal $\mathcal{I}$ such that $\mathcal{O}(\mathcal{G})$ is complete and separated in the topology defined by $\mathcal{I}$ (i.e., $\left.\mathcal{O}(\mathcal{G})=\lim _{\mathcal{O}}(\mathcal{G}) / \mathcal{I}^{m}\right)$, and $\mathcal{O}(\mathcal{G}) / \mathcal{I}=\mathcal{O}(G)$. Finally, we have a supercocommutative coproduct $\Delta: \mathcal{O}(\mathcal{G}) \rightarrow \mathcal{O}(\mathcal{G}) \widehat{\otimes} \mathcal{O}(\mathcal{G})$, where the latter completed tensor product is $\lim _{(}\left(\mathcal{O}(\mathcal{G}) / \mathcal{I}^{m} \otimes \mathcal{O}(\mathcal{G}) / \mathcal{I}^{m}\right)$, defining a topological Hopf algebra structure on $\mathcal{O}(\mathcal{G})$, such that $\mathcal{I}$ is a Hopf ideal, and the isomorphism $\mathcal{O}(\mathcal{G}) / \mathcal{I} \rightarrow \mathcal{O}(G)$ is a Hopf superalgebra isomorphism. 
Let $\mathcal{G}$ be an affine supergroup scheme. The ordinary commutative Hopf algebra $\overline{\mathcal{O}(\mathcal{G})}$ is isomorphic to $\mathcal{O}(G)$ for some affine group scheme $G$, which is referred to as the even part of $\mathcal{G}$.

Let $\mathcal{G}$ be an affine supergroup scheme over $k$, or, more generally, a formal supergroup over $k$, and let $\operatorname{Rep}(\mathcal{G})$ denote the category of finite-dimensional algebraic representations of $\mathcal{G}$ in SuperVect over $k$. Then $\operatorname{Rep}(\mathcal{G})$ is a $k$-linear abelian symmetric rigid tensor category with $\operatorname{End}(\mathbf{1})=k$, where $\mathbf{1}$ denotes the unit object, which admits a fiber functor (= a symmetric tensor functor) to the full $k$-linear abelian symmetric rigid tensor subcategory of SuperVect whose objects are the finite-dimensional supervector spaces. Just like in the even case, $\operatorname{Rep}(\mathcal{G})$ is equivalent to $\operatorname{Corep}(\mathcal{O}(\mathcal{G}))$.

6.3. The proof of Theorem 2.7. Let $\mathcal{G}$ be an affine supergroup scheme over $k$, let $\mathcal{H}:=\mathcal{O}(\mathcal{G})$ be the commutative Hopf superalgebra representing the group functor $\mathcal{G}$, and let $\mathcal{H}^{\prime}$ be the ordinary Hopf algebra associated with $\mathcal{H}$. Then the quotient $\mathcal{H}^{\prime} /\left(k\left[\mathbb{Z}_{2}\right] \ltimes J(\mathcal{H})\right)$ is a commutative Hopf algebra representing the group functor $\mathbb{Z}_{2} \times G$, where $G$ is the even part of $\mathcal{G}$. Therefore $\operatorname{Corep}\left(\mathcal{H}^{\prime} /\left(k\left[\mathbb{Z}_{2}\right] \ltimes J(\mathcal{H})\right)\right)$ is virtually indecomposable by Theorem 2.3, and it follows from Theorems 2.2 and 6.1 that $\operatorname{Corep}(\mathcal{H})$ is virtually indecomposable, as claimed.

For formal supergroups $\mathcal{G}$ over $k$ the proof is completely parallel using Theorem 2.4 about formal groups over $k$.

\section{Acknowledgments}

The author is grateful to J-P. Serre for sending him his proof of Theorem 1.1, for suggesting Question 1.1, and for helpful comments.

The author is indebted to P. Etingof for his help with the proofs, and for his interest in the paper and encouragement.

The author thanks V. Ostrik for telling him about the paper [R].

The research was partially supported by The Israel Science Foundation (grant No. $317 / 09)$.

\section{References}

[A] E. Abe, Hopf algebras, Translated from the Japanese by Hisae Kinoshita and Hiroko Tanaka. Cambridge Tracts in Mathematics, 74, Cambridge University Press, Cambridge, New York, 1980, xii+284pp.

[AEG] N. Andruskiewitsch, P. Etingof and S. Gelaki, Triangular Hopf algebras with the Chevalley property, Michigan J. Math. 49 (2001), 277-298.

[B] H. Boseck, Affine lie supergroups, Math. Nachr. 143 (1989), 303-327.

[C] C. Chevalley, Théorie des Groupes de Lie, III, Hermann, Paris, 1954.

[D] P. Deligne, Catégories tensorielles, (French) [Tensor categories] Dedicated to Yuri I. Manin on the occasion of his 65th birthday, Mosc. Math. J. 2(2) (2002), 227-248.

[DM] P. Deligne and J. Milne, Tannakian categories, Lecture Notes in Mathematics 900 (1982), $101-228$.

[E] P. Etingof, Tensor categories, http://www-math.mit.edu/ etingof/tenscat1.pdf

[EG] P. Etingof and S. Gelaki, Quasisymmetric and unipotent tensor categories, Math. Res. Lett. 15(5) (2008), 857-866.

[K] I. Kaplansky, Fields and rings, Chicago Lectures in Math., Univ. of Chicago Press, Chicago, IL., 1969.

[P] D. Passman, Idempotents in group rings, Proc. Amer. Math. Soc. 28 (1971), 371-374.

[R] R. Rouquier, Familles et blocs d'algèbres de Hecke, C. R. Acad. Sci. Paris t. 329, Série I, 1999, 1037-1042. 
[Se] J.-P. Serre, Lectures on $N_{X}(p)$, to appear.

[Sp] T. A. Springer, Linear algebraic groups, 2nd edition, 'Progress in Mathematics', 9, Birkhauser Boston, Inc., Boston, MA, 1998, xiv+334pp.

[W] W. Waterhouse, Introduction to affine group schemes, 'Graduate Texts in Mathematics', 66, Springer-Verlag, New York, Berlin, 1979, xi+164pp.

Department of Mathematics, Technion-IsRael Institute of Technology, Haifa 32000 , ISRAEL

E-mail address: gelaki@math.technion.ac.il 
